\title{
Outcomes following operative and non-operative management of humeral midshaft fractures: a prospective, observational cohort study of 47 patients
}

\author{
J. J. van Middendorp $\cdot$ F. Kazacsay $\cdot$ P. Lichtenhahn • \\ N. Renner · R. Babst • G. Melcher
}

Received: 12 August 2010/ Accepted: 3 March 2011/Published online: 1 April 2011

(C) The Author(s) 2011. This article is published with open access at Springerlink.com

\begin{abstract}
Background Although the non-operative management of closed humeral midshaft fractures has been advocated for years, the increasing popularity of operative intervention has left the optimal treatment choice unclear.

Objective To compare the outcomes of operative and non-operative treatment of traumatic closed humeral midshaft fractures in adult patients.

Methods A multicentre prospective comparative cohort study across 20 centres was conducted. Patients with AO type $12 \mathrm{~A} 2, \mathrm{~A} 3$ and $\mathrm{B} 2$ fractures were treated with a functional brace or a retrograde-inserted unreamed humeral nail. Follow-up measurements were taken at 6,12 and 52 weeks after the injury. The primary outcome was fracture healing after 1 year. Secondary outcomes included subitems of the Constant score, general patient satisfaction,
\end{abstract}

\author{
J. J. van Middendorp \\ Department of Orthopaedics, Radboud University Nijmegen \\ Medical Center, Nijmegen, The Netherlands \\ e-mail: jvanmiddendorp@gmail.com \\ J. J. van Middendorp \\ Institute of Health and Biomedical Innovation, Queensland \\ University of Technology, Kelvin Grove, Queensland, Australia \\ J. J. van Middendorp \\ Trauma Service, Princess Alexandra Hospital, Brisbane, \\ Queensland, Australia \\ F. Kazacsay \\ Department of Traumatology, Borsod-Abaúj-Zemplén County \\ Teaching Hospital, Miskolc, Hungary \\ e-mail: kazacsay.trauma@bazmkorhaz.hu \\ P. Lichtenhahn \\ Department of Orthopaedics, Spital Schwyz, \\ Schwyz, Switzerland \\ e-mail: pierre.lichtenhahn@spital-schwyz.ch
}

complications and cost-effectiveness parameters. Functions of the uninjured extremity were used as reference parameters. Intention-to-treat analysis was applied with the use of $t$-tests, Fisher's exact tests, Mann-Whitney $U$-tests and adjusted analysis of variance (ANOVA).

Results Forty-seven patients were included. The patient sample consisted of 23 women and 24 men, with a mean age of 52.7 years (range 17-86 years). Of the 47 cases, 14 were treated non-operatively and 33 operatively. The follow-up rate at 1 year was $81 \%$. After 1 year, 11 fractures $(100 \%)$ healed in the non-operative group and at least 24 fractures $(\geq 89 \%)$ healed in the operative group [1 non-union patient (4\%) and no data for 2 patients (7\%)]. There were no significant differences in pain, range of motion (ROM) of the shoulder and elbow, and return to work after 6 weeks, 12 weeks and 1 year. Although operatively treated patients

\author{
N. Renner \\ Department of Traumatology, Kantonsspital Aarau, \\ Aarau, Switzerland \\ e-mail: nrenner@aotrauma.org \\ R. Babst \\ Department of Traumatology, Kantonsspital Luzern, \\ Luzern, Switzerland \\ e-mail: reto.babst@ksl.ch \\ G. Melcher $(\bowtie)$ \\ Department of Surgery, Spital Uster, \\ Brunnenstrasse 42, 8610 Uster, Switzerland \\ e-mail: gian.melcher@spitaluster.ch
}


showed significantly greater shoulder abduction strength ( $p=0.036)$, elbow flexion strength $(p=0.021)$, functional hand positioning $(p=0.008)$ and return to recreational activities ( $p=0.043)$ after 6 weeks, no statistically significant differences existed in any outcome measure at the 1-year follow-up.

Conclusions Our findings indicate that the non-operative management of humeral midshaft fractures can be expected to have similar functional outcomes and patient satisfaction at 1 year, despite an early benefit to operative treatment. If no radiological evidence of fracture healing exists in nonoperatively treated patients during early follow-up, a switch to surgical treatment results in good functional outcomes and patient satisfaction.

Keywords Humeral shaft fracture .

Non-operative treatment - Functional brace .

Operative treatment · Unreamed humeral nail (UHN) .

Prospective $\cdot$ Cohort study

\section{Introduction}

Fractures of the humerus account for approximately 5-7\% of all fractures [1, 2]. The relative incidences of the proximal, diaphyseal and distal humeral fractures are approximately 40, 20 and 40\%, respectively [1, 3, 4]. Similar to proximal and distal humeral fractures [5-8], the management of diaphyseal (midshaft) humeral fractures has also been a lively topic of debate.

While closed humeral midshaft fractures can be managed non-operatively with reported union rates as high as 94\% [9], the operative management of these fractures has become popular over the last two decades $[10,11]$. In particular, non-operatively treated transverse and short oblique midshaft fractures have been associated with delayed union in reference works $[12,13]$. These fracture subtypes are, therefore, considered to benefit from a surgical treatment. Nonetheless, consensus regarding the optimal treatment of oblique, transverse and bending wedge fractures of the humeral midshaft is lacking.

Proponents of operative fixation believe that immediate stable fixation of the humerus allows for a rapid return to normal function and more predictable alignment and fracture healing. However, those who argue in favour of non-operative approaches cite the increased risks of operation, including infections and radial nerve injuries. Despite these important contentious issues, the number of comparative studies evaluating alternative approaches in the management of humeral midshaft fractures is scarce. In fact, to our knowledge, no prospective cohort study comparing non-operative and operative treatment for humeral midshaft fractures has been published to date.
The aim of this prospective study was to compare the outcomes of operative and non-operative treatment techniques applied to oblique, transverse and bending wedge fractures of the humeral midshaft. Based on the previous literature, we hypothesised that non-operative management would result in comparable treatment outcomes to operative treatment at 1 year [14-16].

\section{Methods}

From October 2000 to May 2004, a prospective multicentre comparative cohort study was conducted across 20 rural and referral university trauma centres situated in Switzerland (18), Austria (1) and Hungary (1). Although this study initially begun as a randomised controlled study, the study design had to be adjusted to a non-randomised prospective comparative study because of difficulties experienced in the treatment allocation process. The number of treating physicians at each centre varied from one to four. Patients with a traumatic closed humeral midshaft fracture (transverse or short oblique) were included and treated nonoperatively or operatively according to the physician's decision. Radiographic and functional status data were prospectively collected during initial hospital admission and 6,12 and 52 weeks after the injury. The study protocol was approved by the local ethics committees and the subjects gave their written informed consent before participating in the study. The study was conducted according to the principles of good clinical practice and the Declaration of Helsinki [17].

\section{Patient eligibility}

Eligible patients were included in the study when they met the following criteria: (1) diagnosed with a traumatic humeral midshaft fracture classified as AO type $12 \mathrm{~A} 2,12$ $\mathrm{A} 3$ or $12 \mathrm{~B} 2$ based on conventional anteroposterior and lateral radiographs [18]; (2) over 16 years of age and in full possession of their mental faculties; (3) 7 days or less between injury and the start of treatment; (4) likely or could guarantee to be available for follow-up visits up to the end of the study; and (5) gave written informed consent. Patients with the following condition were excluded: (1) soft tissue injury: open fracture type Gustilo-Anderson Type II or higher [19]; (2) pre-existing humeral pseudarthrosis; (3) pathological fracture and/or re-fracture; (4) additional proximal or distal humeral fracture; (5) patients with pre-existing medical conditions that would predictably have a negative prognostic effect on treatment outcomes (e.g. congenital bone and connective tissue disorders, any type of cancer, neurological disorders etc.); and (6) bilateral humeral shaft fracture. 
Management

Since a non-randomised study design was applied, it was up to the treating physician to determine the treatment of choice, i.e. non-operative or operative treatment. Nonoperative treatment consisted of an initial temporary brace of choice until the reduction of local post-traumatic swelling. Once the reduction of swollen soft tissue was acceptable according to the treating physician, a functional brace analogous to the Sarmiento principle was placed [20]. As multiple factors potentially influence the timing of fracture healing, the duration of non-operative treatment was not standardised [21]. The number of outpatient physiotherapy visits was also not standardised. Instead, the presence of preexisting co-morbidities was documented and the number of physiotherapist visits was applied as a descriptive outcome measure. Patients' adherence to the non-operative treatment protocol was not quantitatively assessed.

Operative treatment consisted of a retrograde placed locked unreamed intramedullary humeral nail (UHN). A small amount of eligible patients were treated with antegrade placed locked UHN or plate fixation; however, these were not included in the analysis (Fig. 1). In order to achieve a homogeneous cohort, only patients treated with a retrograde placed locked UHN were included in the analysis. The retrograde intramedullary nailing procedure was standardised and performed according to the AO principles of fracture management [22]. The duration of surgery was recorded for each patient and the use of post-operative immobilisation was not standardised.

\section{Outcome assessment}

The outcomes for operative and non-operative treatment were assessed after 6, 12 and 52 weeks (Fig. 1). The primary outcome of this study was fracture healing after 1 year. Fracture healing was defined as the presence of three or four cortices of the humerus with callus formation as shown on lateral and anteroposterior radiographs [23]. The presence or absence of fracture healing was determined by the treating specialist.

Secondary outcomes included sub-items of the Constant score, general patient satisfaction, complications and costeffectiveness parameters. Functional outcomes for operative and non-operative treatment were assessed using the Constant scoring system [24]. This system has been shown to have excellent reliability and responsiveness, and has been adopted by the European Society for Shoulder and Elbow Surgery (ESSES) [25]. This scoring system consists of four variables that are used to assess the function of the shoulder. The right and left shoulders are assessed separately. It consists of two physician-assessed variables (range of motion [ROM] and strength) and two patient-reported variables (pain and activities in daily living [ADL], including sleep, work, recreation/sport) [24]. The two physician-assessed variables were completed by the treating physician. The
Fig. 1 Flowchart of included patients with subsets of followup measurements
Patient recruitment
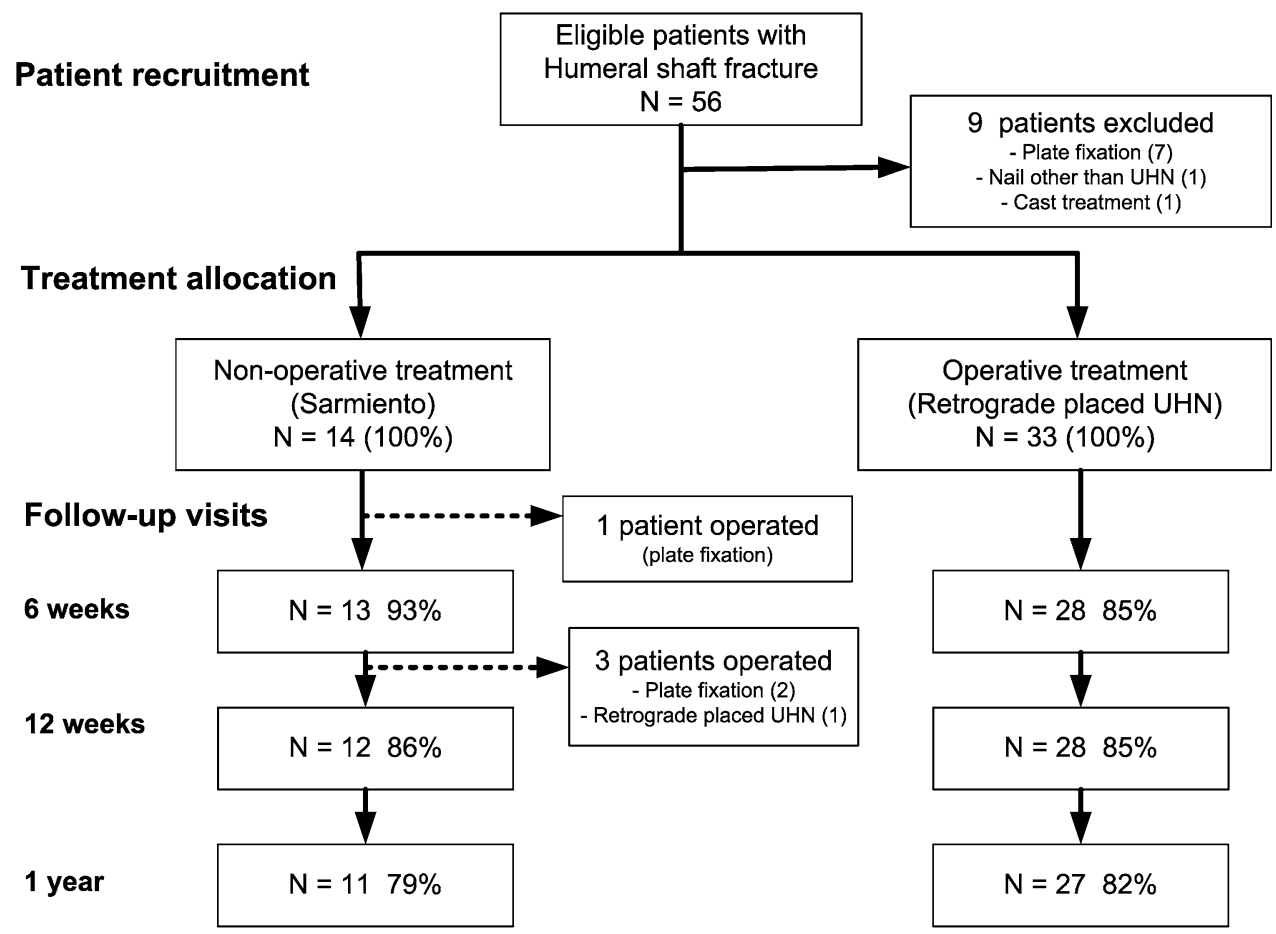

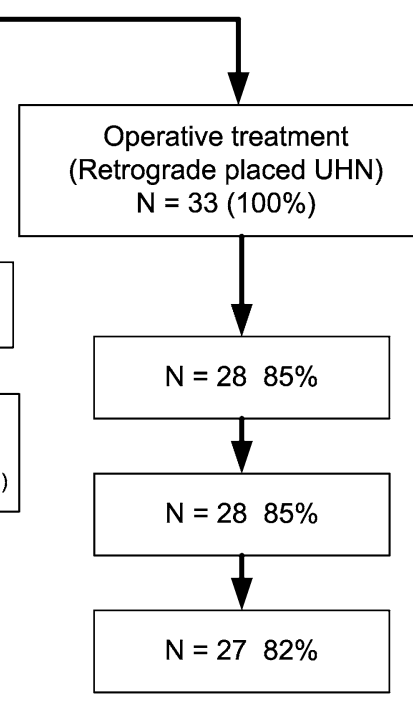

UHN : Unreamed Humeral Nail 
Table 1 A summary of baseline demographics and clinical characteristics of included patients $(n=47)$

\begin{tabular}{lll}
\hline Characteristic & $\begin{array}{l}\text { Non-operative } \\
\text { group }(n=14)\end{array}$ & $\begin{array}{l}\text { Operative } \\
\text { group }(n=33)\end{array}$ \\
\hline Baseline demographics & $9(64)$ & $14(42)$ \\
Female sex, no. (\%) & $51( \pm 24 ; 17-84)$ & $53( \pm 19 ; 17-86)$ \\
Age, years (SD; range) & $1(7)$ & $1(3)$ \\
Diabetes mellitus type II, no. (\%) & $1(7)$ & $1(3)$ \\
Obesity (BMI > 30), no. (\%) & $1(7)$ & $2(6)$ \\
Medication for osteoporosis, no. (\%) & & \\
Injury characteristics & $6(43)$ & $6(18)$ \\
AO Classification [13], no. (\%) & $5(36)$ & $17(52)$ \\
12 A2 & $3(21)$ & $10(30)$ \\
12 A3 & $0(0)$ & $2(6)$ \\
12 B2 & $1(7)$ & $2(6)$ \\
Gustilo-Anderson Type I [8], no. (\%) & $1(7)$ & $6(18)$ \\
Radial nerve injury, no. (\%) & & $89( \pm 40 ; 35-190)$ \\
Fracture other extremity, no. (\%) & - & $12(36)$ \\
Treatment characteristics & - &
\end{tabular}

ROM was examined according to the AO Neutral- 0 method [26]. We present each Constant score category separately rather than as an agregate score in order to enable possible specific differences in operative and non-operative treatments to be studied. Additionally, the strength and ROM of the ipsilateral and contralateral elbows were also assessed by applying the same principles as the shoulder examination.

Complications and cost-effectiveness parameters including the duration of hospital admission and the number of outpatient physiotherapy visits were reported prospectively. Patients' overall treatment satisfaction was assessed with the use of the visual analogue scale (VAS). In addition, patients' satisfaction of the following more specific domains were also assessed: cosmetic appearance, function and pain.

Data analysis

Statistical analyses were performed using STATA, version 10.0 (Stata Corporation, College Station, Texas, USA). All data processing and analyses were conducted independently of the participating physicians. We summarised continuous data with means, medians, ranges and standard deviations (SDs). If the data were skewed, we presented median values for both groups. The two groups were compared with regards to continuous and categorical outcomes using the non-parametric Mann-Whitney $U$-test and Fisher's exact test, respectively. Ordered categories were analysed with the Mann-Whitney $U$-test. Comparison of continuous outcomes (e.g. ROM parameters) between the injured and the contralateral uninjured side at each follow-up examination was implemented using the Wilcoxon signed-rank test (paired data). The longitudinal data were compared using analysis of variance (ANOVA) for repeated measures, adjusted for putative confounding baseline demographics and clinical characteristics as specified at each separately presented adjusted ANOVA analysis. All tests were two-tailed and we considered the conventional level of statistical significance as $p<0.05$.

\section{Results}

During a 4-year period, 47 patients with humeral midshaft fractures in 20 hospitals were included in the study (Fig. 1). A summary of the baseline demographics and clinical characteristics of the study groups is presented in Table 1 . The mean patient age at the time of injury was 52.7 years (range 17-86 years). Of the 47 humeral midshaft fractures, 14 were treated non-operatively and 33 were treated operatively. The most common fracture pattern was AO type 12 A3 (47\%). Seven patients had additional long bone fractures, six of whom were treated operatively. Two operatively treated patients had a Gustilo-Anderson Type I open fracture.

Four of the 14 non-operatively treated patients switched to operative treatment during follow-up. Due to low treatment compliance, one 85-year-old female patient preferred surgical treatment 2 days after the start of nonoperative treatment (Fig. 1). The intention-to-treat principle was applied to all of these four patients. The overall follow-up at 12 months was $81 \%$. In the non-operative 
Fig. 2 Range of motion (ROM) at 6 weeks: (1) shoulder flexion arch, (2) shoulder abduction arch and (3) elbow flexionextension arch in proportions of categorised values

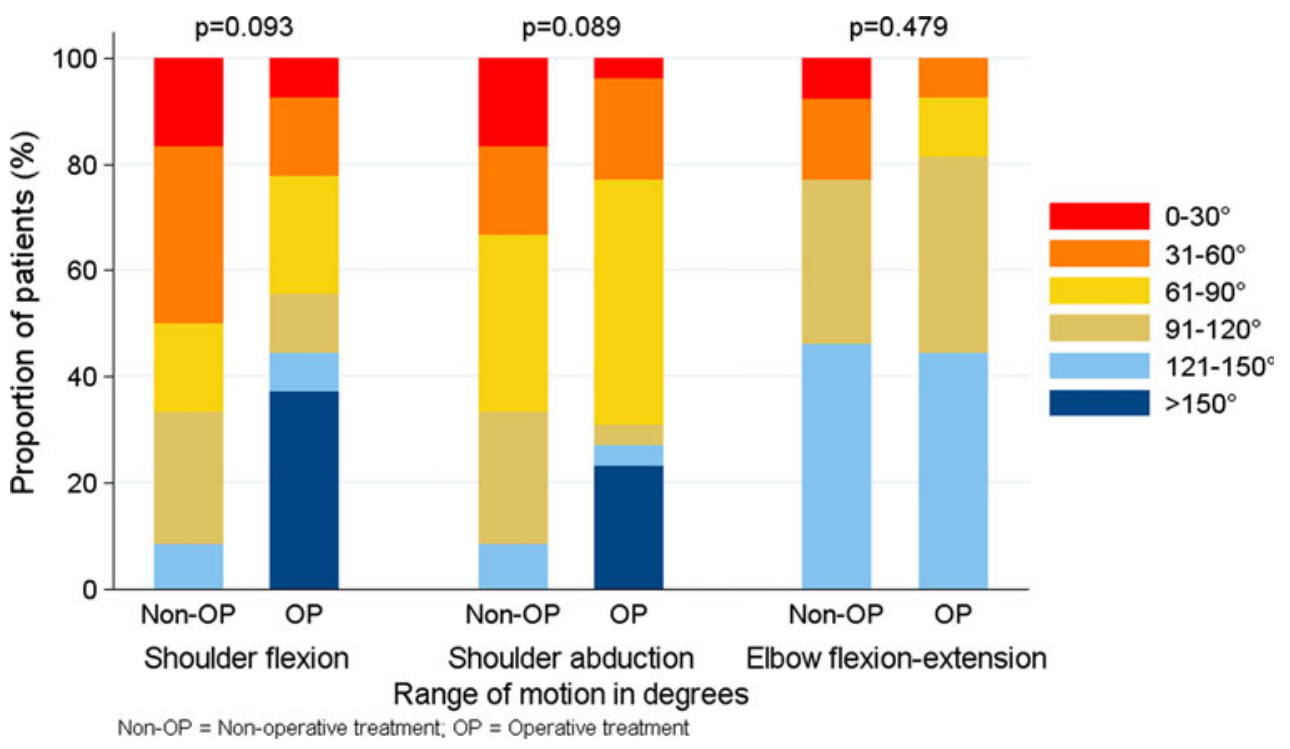

group, 11 out of 14 patients completed 1 year of follow-up $(79 \%)$; in the operative group, this figure was 27 out of 33 $(82 \%)$.

Fracture healing

In the non-operatively treated patients, 11 (100\%) fractures were healed 1 year after the injury. A total of 3 out of 11 non-operatively treated patients showed insufficient callus formation during early follow-up ( $<8$ weeks) and these patients switched to surgical treatment (Fig. 1). In the operative group, at least 24 fractures in 27 patients $(\geq 89 \%)$ were healed after 1 year. Despite the presence of callus formation, one patient had no radiographic evidence of stable fracture healing after 1 year. Although this 52-yearold female patient experienced severe pain during followup (VAS score 2), she refused any further treatment. Two other patients did not undergo radiographic assessment after 1 year and, therefore, their fracture healing status could not be determined.

\section{Constant score: ROM and strength}

Compared to the function of the uninjured upper extremity, both non-operatively and operatively treated patients had a statistically significant reduced ROM of the shoulder flexion arch ( $p=0.002$ and $p=0.001$, respectively), shoulder abduction arch $(p=0.002$ and $p<0.001$, respectively) and elbow flexion-extension arch ( $p=0.008$ and $p<0.001$, respectively) at 6 weeks follow-up. No statistically significant differences in the range of the elbow flexion-extension, shoulder flexion and shoulder abduction arch were observed between the non-operatively and operatively treated patients at 6 weeks, 12 weeks and 1 year. After categorisation into six categories of ROM $\left(0-30^{\circ}, 31-60^{\circ}, 61-90^{\circ}, 91-120^{\circ}, 121-150^{\circ}\right.$ and $\left.>150^{\circ}\right)$, operatively treated patients had a tendency to have greater range of the flexion ( $p=0.093)$ and abduction $(p=0.089)$ of the injured shoulder than non-operative patients at 6 weeks (Fig. 2). However, this difference was no longer evident 1 year after the injury.

Relative shoulder abduction strength was significantly greater in operatively treated patients than those treated nonoperatively at 6 weeks post-injury $(p=0.036)$ (Fig. 3). Compared to only one out of the non-operatively treated patients $(9 \%)$, almost 1 in 4 operatively treated patients (24\%) already had symmetrical power to their uninjured side at 6 weeks. Strength differences were no longer apparent by 12 weeks and 12 months post-injury.

At the 6-week follow-up, the relative elbow flexion strength was also significantly greater in operatively treated patients than in non-operative patients ( $p=0.021)$ (Fig. 3). Similarly, strength differences were no longer apparent by 12 weeks and 12 months post-injury. Although there was a tendency towards an increased relative elbow extension strength in operatively treated patients at 6 weeks $(p=0.071$, ANOVA), no statistically significant differences between the two cohorts were identified at any follow-up time.

Constant score: pain and ADL

At 6 weeks, 3 months and 12 months, no significant differences in shoulder pain across patients treated nonoperatively or operatively were found. In contrast, the ADL hand positioning of the injured extremity was significantly diminished in the non-operative group compared to the operative group at 6 weeks $(p=0.008)$ (Fig. 4). At 12 weeks and at 1 year after the injury, however, this difference was no longer evident. Among 38 patients (11 non-operative, 27 operative), $30(79 \%)$ had returned to 
Fig. 3 Shoulder abduction and elbow flexion strength of injured extremity compared to uninjured extremity
Fig. 4 Scoring for activities in daily life (ADL): positioning of the hand according to the Constant score

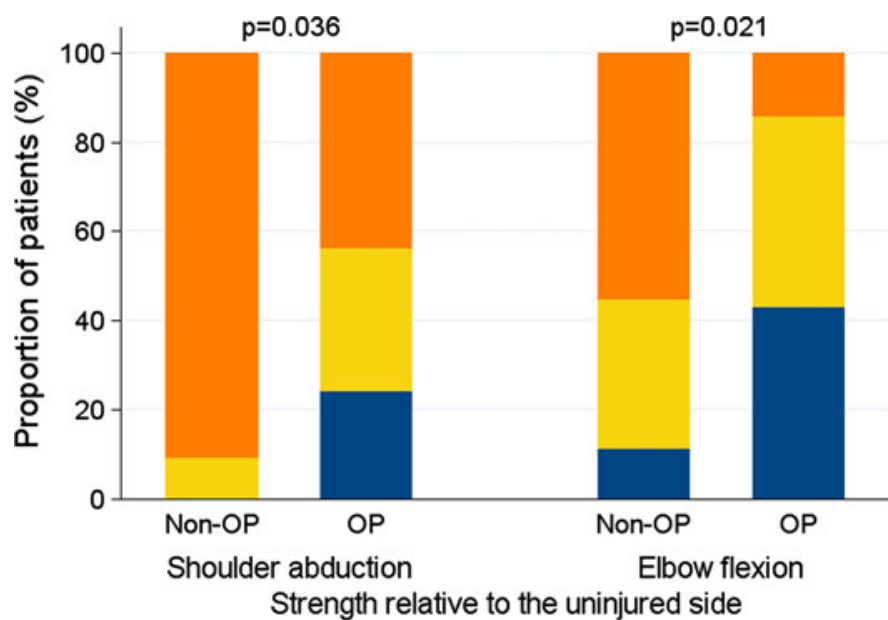

Non-OP = Non-operative treatment; $\mathrm{OP}=$ Operative treatment

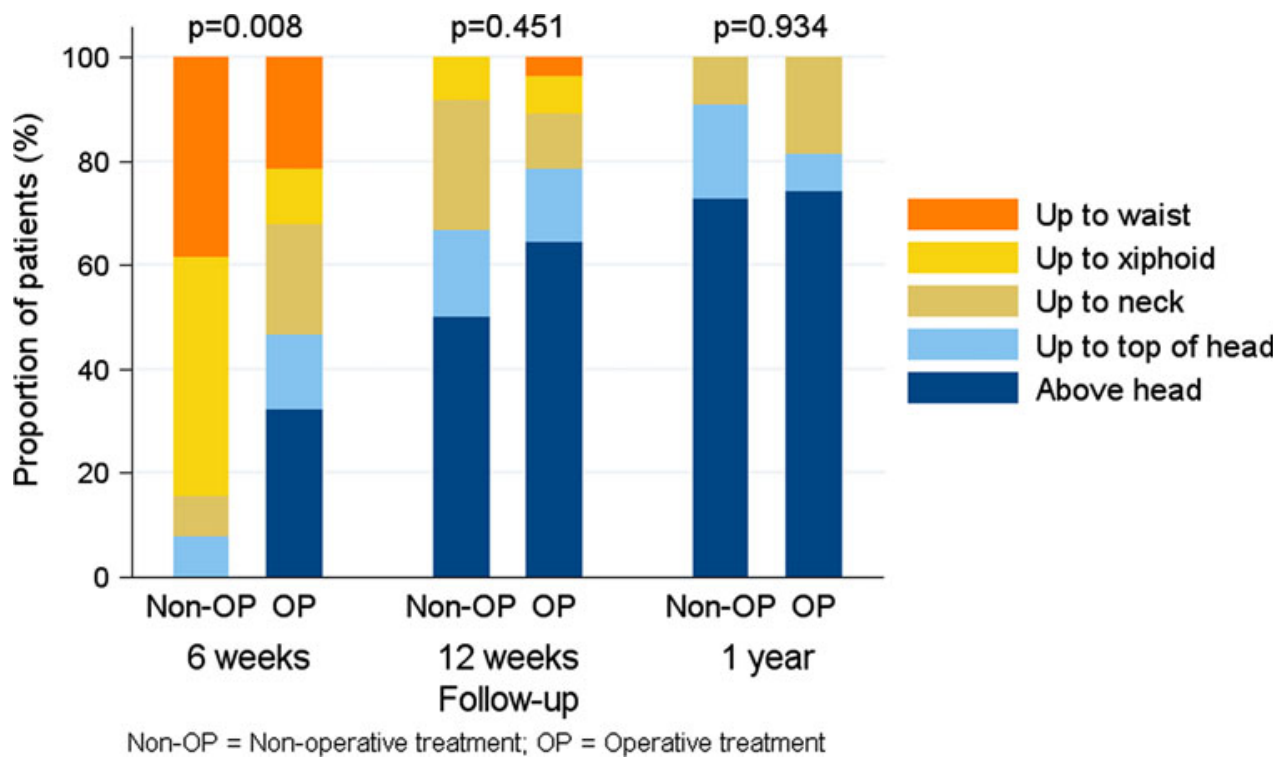

work without restrictions after 12 months. No statistically significant differences between the two cohorts were identified regarding return to work at any follow-up time. However, operatively treated patients returned to recreation activities earlier than non-operatively treated patients at 6 weeks ( $p=0.043$, Mann-Whitney $U$-tests). At 12 weeks and at 1 year, the proportion of patients returning to unrestricted recreational activities were quite similar across the two groups (Fig. 5).

\section{Complications}

No treatment-related complications were reported in the non-operative treatment group. All concomitant radial nerve injuries recovered completely in both the non-operative and operative treatment groups. Six treatment-related complications occurred in 5 of 33 operatively treated patients $(15 \%)$. A longitudinal fissure developed during insertion of the UHN in one 61-year-old male patient with a closed, isolated 12 A2 fracture. According to the treating physician, the development of the fissure can be attributed to an exceptionally narrow medullar cavity. One other patient showed evidence of rotator cuff injury as confirmed on magnetic resonance imaging (MRI). It was not entirely clear whether the rotator cuff injury was a concomitant traumatic injury or a pre-existing lesion. The UHN was removed 10 months after the initial date of surgery. Another patient experienced discomfort and had a limited ROM of the ipsilateral shoulder 5 months after the injury. MRI did not show evidence of a rotator cuff injury. Consequently, an arthroscopic arthrolysis and an additional subacromial decompression were performed together and the locking screw was removed. In one 61-year-old female patient, a loosening of a distal locking screw was observed after 14 weeks and successful fracture healing. At this moment, the patient also showed a radial nerve palsy at the 
Fig. 5 Scoring for ADL: return to recreational activities according to the Constant score

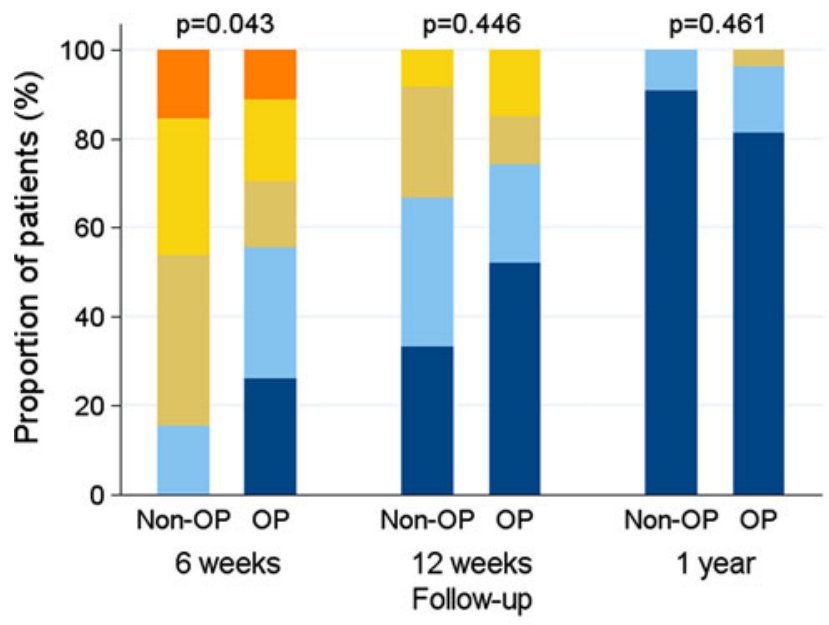

Non-OP = Non-operative treatment; $\mathrm{OP}=$ Operative treatment

Table 2 Patients' overall treatment satisfaction at 1-year follow-up, including specific domains: cosmetic appearance, function and pain with the use of a visual analogue scale (VAS) scale $(0=$ unsatisfied, $10=$ satisfied $)$

\begin{tabular}{|c|c|c|c|c|c|}
\hline \multirow[t]{2}{*}{ VAS satisfaction } & \multicolumn{2}{|c|}{ Non-operative group $(n=11)$} & \multicolumn{2}{|c|}{ Operative group $(n=26)$} & \multirow{2}{*}{$\begin{array}{l}\text { Mann-Whitney } U \text {-test } \\
p \text {-value }\end{array}$} \\
\hline & Mean & SD & Mean & SD & \\
\hline Overall & 9.7 & 0.5 & 9.0 & 1.8 & 0.435 \\
\hline Cosmetic & 9.5 & 0.8 & 9.3 & 1.2 & 0.835 \\
\hline Function & 9.2 & 1.3 & 9.3 & 1.3 & 0.689 \\
\hline Pain & 9.7 & 0.6 & 9.0 & 2.1 & 0.371 \\
\hline
\end{tabular}

ipsilateral extremity, resulting in severe functional disability. A reasonable explanation for this (secondary) nerve palsy was not found. More information on this patient is not known because she did not attend the last follow-up. One post-operative superficial wound infection was reported, which was successfully treated with the use of antibiotics alone.

Cost-effectiveness parameters and treatment satisfaction

After adjustments for recorded additional injuries and preexisting medical conditions, there was a statistically nonsignificant tendency towards an increased duration of hospital stay in patients treated operatively (median: 7.3 days, SD: 4.3, range: 6-21 days) compared to patients treated non-operatively (median: 5.2 days, SD: 4.0, range: 5-15 days), $p=0.088$. The mean number of outpatient physiotherapy visits were similar across the non-operative and operative groups (12 vs. 13 visits, respectively, $p=$ 0.779, ANOVA).

With the exclusion of ten retired patients (two nonoperative and eight operative), there was no statistically significant difference in the duration of occupational disablement between the two treatment groups $(p=0.697$, Fisher's exact test). At the final 12-month follow-up, patients were equally satisfied with their treatment results in both the non-operative and operative groups. In addition, no significant differences were observed in the cosmetic appearance, function and pain satisfaction domains (Table 2).

\section{Discussion}

In this prospective cohort study of 47 patients with humeral midshaft fractures with $81 \%$ follow-up to 1 year, we report the following: (1) operative treatment with UHN resulted in greater strength, functional hand positioning and earlier return to recreational activities at 6 weeks; (2) any advantages to operative treatment were no longer apparent at 12 weeks and at 1 year; (3) operative treatment tended to result in longer hospital stays and resulted in more treatment-related complications; (4) if no radiological evidence of fracture healing existed in non-operatively treated patients during early follow-up, a switch to surgical treatment resulted in good functional outcomes and patient satisfaction.

Our findings support non-operative management as a viable contemporary option for the management of even transverse and short oblique closed fractures of the humeral midshaft. Papasoulis et al. [9] recently reviewed the radiographic and functional outcomes of non-operatively 
treated humeral shaft fractures. It was concluded that the functional bracing of closed humeral shaft fractures results in high fracture consolidation rates, good functional outcomes and reduced health care expenditures compared to surgical treatment. Moreover, with the choice of functional bracing treatment possible adverse events as a result of operative treatment can be avoided. In this study, six complications related to operative treatment were reported, of which one resulted in permanent physical impairment and two required a re-operation.

Advocates of the surgical treatment of closed humeral midshaft fractures claim that operative stabilisation achieves better alignment of the humeral shaft than nonoperative treatment. Although malalignment, or residual deformity, was not applied as an outcome measure in this study, functional outcomes and the cosmetic appearance of non-operatively treated patients were satisfactory. In a large patient series, Sarmiento et al. [15] reported satisfactory functional and cosmetic outcomes in patients with a residual varus-valgus or anteroposterior angular deformity of more than $10^{\circ}$ after the non-operative treatment of humeral shaft fractures. The generally good outcomes with non-operative treatment may be due, in part, to the tolerance of malunion in the arm. Papasoulis et al. [9] summarised that an angular deformity up to $20^{\circ}$ is well tolerated by the musculature around the humerus. In addition, the humerus can tolerate up to $2 \mathrm{~cm}$ of shortening as a result of over-riding fracture fragments with little functional deficit.

A number of randomised trials comparing various types of operative treatments for diaphyseal fractures of the humerus have been published in the last decade $[10,11,27$, 28]. Unfortunately, no randomised controlled trial comparing non-operative and operative treatment for humeral shaft fractures has been published to date. To our knowledge, this is the first published prospective cohort study comparing non-operative to operative treatment of transverse and short oblique humeral midshaft fractures. Only two retrospective studies comparing functional bracing and operative stabilisation for the treatment of humeral shaft fractures have been published to date. Klestil et al. [29] conducted a retrospective study comparing operative and non-operative management in 63 patients with humeral shaft fractures. Conservative treatment was found to be at least equivalent to, if not better than, the operative option. Comparable conclusions were drawn by Wallny et al. [30] in a retrospective analysis of 89 patients treated with a functional brace or retrograde placed humerus nail.

Given the current level of evidence and the continuing debate over the optimal treatment choice for closed humeral shaft fractures, future randomised trials comparing nonoperative to operative treatments are certainly warranted. However, although the need for randomised clinical trials has been repeatedly expressed, we would like to stress the difficulties that can be faced during their preparation and conduct. Our study was initially begun as a randomised controlled study. It was apparent soon after its start that the majority of collaborating physicians did not adhere to the randomised treatment allocation procedure as they had a strong preference for the surgical treatment option. Consequently, the study design had to be adjusted to a non-randomised prospective comparative study. In future, this treatment preference issue may be solved by applying an alternative study design: the expertise-based randomised controlled trial (EBT) [31]. In an EBT, participants are randomised to clinicians with expertise in one of the specific interventions. Consequently, physicians may be more willing to participate because they only have to perform the procedure which they have developed a preference for and/ or expertise. Nonetheless, one should bear in mind that, in contrast to an elective setting, the implementation of such a study design will not always be feasible in a trauma setting with multiple care providers on different calls.

Despite our switch from a randomised trial into a nonrandomised prospective cohort study, we still experienced difficulties in recruiting a satisfactory number of patients. With 47 traumatic humeral midshaft fracture patients included in 20 centres during a 3.5-year period, this study clearly illustrates that patient recruitment for prospective (randomised) controlled studies remains one of the major challenges in clinical orthopaedic trauma research.

Three non-operatively treated patients showed early radiographic evidence of fracture non-union and were subsequently operated on successfully. In contrast to these findings, Ekholm et al. [32] reported worse functional outcomes after revision surgery for humeral shaft fracture patients with fracture non-union after initial functional bracing treatment. All three early non-union patients in this study were female, older than 50 years of age and had an AO fracture type $12 \mathrm{~A} 2$. This is consistent with factors associated with humeral shaft fracture non-union reported in the literature. Fracture non-unions are more likely in non-operatively treated oblique AO type A fractures [9, 33]. Whether patients with this specific type of injury benefit from initial operative stabilisation needs to be clarified in future high-volume research. Although patients' adherence to the non-operative treatment regimen was not recorded in this study, treatment non-compliance may be another explanation for the occurrence of non-unions. Noncompliance to functional bracing and severe obesity have been widely regarded as relative contraindications for nonoperative fracture management.

Our study is strengthened by prospective data collection, a high follow-up rate at 12 months and the use of valid and clinically relevant patient outcome measures. Capturing patient satisfaction with the management approaches is another strength of this study. Although not reported, we also 
performed ancillary "as-treated" analyses in addition to reported "intention-to-treat" analyses. However, none of these ancillary analyses showed alterations of (in)significant differences between operatively and non-operatively treated patients with regard to the outcomes under investigation. We are limited, however, by the small sample size that may have left some of our analyses underpowered. Unfortunately, the numbers of ineligible patients have not been recorded in our database. Although the numbers for the study are small, our careful data collection and analysis of outcomes assures the reader that the data we did collect is internally valid and useful for planning future studies. Despite the non-randomised patient selection, the baseline parameters of included patients were remarkably similar in both treatment groups. Besides this, putative confounding factors were recognised and adjusted for by applying ANOVA.

In summary, our findings indicate that the non-operative management of humeral midshaft fractures can be expected to have similar functional outcomes and patient satisfaction at 1 year, despite an early benefit to operative treatment. If no radiological evidence of fracture healing exists in nonoperatively treated patients during early follow-up, a switch to surgical treatment results in good functional outcomes and patient satisfaction.

Acknowledgments This study was financially supported by the AO Alumni Association Schweiz. We are grateful to Dr. Matthias Müller and Mrs. Riitta Schmid for their assistance with the data collection and data processing. We are indebted to Dr. Laurent Audigé for his invaluable assistance with the statistical analyses and preparation of the paper. We also thank Mr. Diarmuid De Faoite for his assistance with the preparation of this manuscript. We thank Dr. Beate Hanson for her thorough and insightful comments on earlier drafts of this manuscript. We would also like to thank the participating physicians and centres who contributed to the data presented in this study: Dr. C. Del Notaro, Limmatspital Zürich, Zürich, Switzerland; Dr. S. Eberdorfer, Wilhelminenspital, Vienna, Austria; Dr. K. Eid, Universitätsspital Zürich, Zürich, Switzerland; Dr. F. Hardegger, Regionalspital Surselva, Ilanz, Switzerland; Dr. R. Kratter, Kantonsspital Münsterlingen, Scherzingen, Switzerland; Dr. P. Messmer, Kantonsspital Basel, Basel, Switzerland; Dr. B. Noesberger, Spital Interlaken, Unterseen, Switzerland; Prof. dr. P. Ochsner, Kantonsspital Liestal, Liestal, Switzerland; Dr. A. Olah, Spital Rorschach, Rorschach, Switzerland; Dr. K. Pieper, Kreisspital Muri, Muri, Switzerland; Dr. V. Rehli, Kantonsspital Walenstadt, Walenstadt, Switzerland; Dr. D. Scharplatz, Krankenhaus Thusis, Thusis, Switzerland; Dr. W. Schweizer, Kantonsspital Schaffhausen, Schaffhausen, Switzerland; Dr. C. Sommer, Kantonsspital Chur, Chur, Switzerland; Dr. A. Villiger, Spital Wetzikon, Wetzikon, Switzerland; and Dr. A. Vollenwelder, Spital Männedorf, Männedorf, Switzerland. J.J. van Middendorp was supported by a fellowship from AO Clinical Investigation and Documentation (AOCID)

Conflict of interest None reported by any of the authors.

Open Access This article is distributed under the terms of the Creative Commons Attribution Noncommercial License which permits any noncommercial use, distribution, and reproduction in any medium, provided the original author(s) and source are credited.

\section{References}

1. Brinker MR, O'Connor DP. The incidence of fractures and dislocations referred for orthopaedic services in a capitated population. J Bone Joint Surg Am. 2004;86(2):290-7.

2. van Staa TP, Dennison EM, Leufkens HGM, Cooper C. Epidemiology of fractures in England and Wales. Bone. 2001;29(6): 517-22.

3. Buhr AJ, Cooke AM. Fracture patterns. Lancet. 1959;1(7072): 531-6.

4. Rose SH, Melton LJ 3rd, Morrey BF, Ilstrup DM, Riggs BL. Epidemiologic features of humeral fractures. Clin Orthop Relat Res. 1982;(168):24-30.

5. Lanting B, MacDermid J, Drosdowech D, Faber KJ. Proximal humeral fractures: a systematic review of treatment modalities. J Shoulder Elbow Surg. 2008;17(1):42-54.

6. Pollock JW, Faber KJ, Athwal GS. Distal humerus fractures. Orthop Clin North Am. 2008;39(2):187-200, vi.

7. Throckmorton TW, Zarkadas PC, Steinmann SP. Distal humerus fractures. Hand Clin. 2007;23(4):457-69, vi.

8. Vallier HA. Treatment of proximal humerus fractures. J Orthop Trauma. 2007;21(7):469-76.

9. Papasoulis E, Drosos GI, Ververidis AN, Verettas DA. Functional bracing of humeral shaft fractures. A review of clinical studies. Injury. 2010;41(7):e21-4.

10. Chapman JR, Henley MB, Agel J, Benca PJ. Randomized prospective study of humeral shaft fracture fixation: intramedullary nails versus plates. J Orthop Trauma. 2000;14(3):162-6.

11. McCormack RG, Brien D, Buckley RE, McKee MD, Powell J, Schemitsch EH. Fixation of fractures of the shaft of the humerus by dynamic compression plate or intramedullary nail. A prospective, randomised trial. J Bone Joint Surg Br. 2000;82(3):336-9.

12. McKee MD. Fractures of the shaft of the humerus. In: Bucholz RW, Heckman JD, Court-Brown CM, Koval KJ, Tornetta P, Wirth MA, editors. Rockwood and Green's fractures in adults. 6th ed. Philadelphia: Lippincott Williams \& Wilkins; 2005. p. $1118-59$.

13. Schatzker J. Fractures of the humerus (12-A, B, and C). In: Schatzker J, Tile M, editors. The rationale of operative fracture care. 3rd ed. Berlin: Springer-Verlag; 2005. p. 91-102.

14. Rommens PM, Blum J, Runkel M. Retrograde nailing of humeral shaft fractures. Clin Orthop Relat Res. 1998;(350):26-39.

15. Sarmiento A, Zagorski JB, Zych GA, Latta LL, Capps CA. Functional bracing for the treatment of fractures of the humeral diaphysis. J Bone Joint Surg Am. 2000;82(4):478-86.

16. Nast-Kolb D, Knoefel WT, Schweiberer L. The treatment of humeral shaft fractures. Results of a prospective AO multicenter study. Unfallchirurg. 1991;94(9):447-54.

17. [No authors listed]. World Medical Association declaration of Helsinki. Recommendations guiding physicians in biomedical research involving human subjects. JAMA. 1997;277(11):925-6.

18. Müller ME, Nazarian S, Koch P. Classification AO des fractures. Tome I. Les os longs. 1st ed. Berlin, Germany: Springer; 1987.

19. Gustilo RB, Anderson JT. Prevention of infection in the treatment of one thousand and twenty-five open fractures of long bones: retrospective and prospective analyses. J Bone Joint Surg Am. 1976;58(4):453-8.

20. Sarmiento A, Kinman PB, Galvin EG, Schmitt RH, Phillips JG. Functional bracing of fractures of the shaft of the humerus. J Bone Joint Surg Am. 1977;59(5):596-601.

21. Gaston MS, Simpson AHRW. Inhibition of fracture healing. J Bone Joint Surg Br. 2007;89(12):1553-60.

22. Rüedi TP, Buckley RE, Moran CG, editors. AO principles of fracture management, vol. 2: specific fractures. 2nd ed. New York: Thieme Verlag; 2007. p. 598-604. 
23. Morshed S, Corrales L, Genant H, Miclau T 3rd. Outcome assessment in clinical trials of fracture-healing. J Bone Joint Surg Am. 2008;90(Suppl 1):62-7.

24. Constant CR, Murley AH. A clinical method of functional assessment of the shoulder. Clin Orthop Relat Res. 1987;(214): $160-4$.

25. Roy JS, MacDermid JC, Woodhouse LJ. A systematic review of the psychometric properties of the Constant-Murley score. J Shoulder Elbow Surg. 2009;19(1):157-64.

26. Ryf C, Weymann A, editors. Range of motion-AO Neutral-0 method. 1st ed. New York: Thieme Verlag; 1999.

27. Cheng HR, Lin J. Prospective randomized comparative study of antegrade and retrograde locked nailing for middle humeral shaft fracture. J Trauma. 2008;65(1):94-102.

28. Putti AB, Uppin RB, Putti BB. Locked intramedullary nailing versus dynamic compression plating for humeral shaft fractures. J Orthop Surg (Hong Kong). 2009;17(2):139-41.
29. Klestil T, Rangger C, Kathrein A, Brenner E, Beck E. The conservative and surgical therapy of traumatic humeral shaft fractures. Chirurg. 1997;68(11):1132-6.

30. Wallny T, Sagebiel C, Westerman K, Wagner UA, Reimer M. Comparative results of bracing and interlocking nailing in the treatment of humeral shaft fractures. Int Orthop. 1997;21(6): 374-9.

31. Devereaux PJ, Bhandari M, Clarke M, Montori VM, Cook DJ, Yusuf S, Sackett DL, Cinà CS, Walter SD, Haynes B, Schünemann HJ, Norman GR, Guyatt GH. Need for expertise based randomised controlled trials. BMJ. 2005;330(7482):88.

32. Ekholm R, Tidermark J, Törnkvist H, Adami J, Ponzer S. Outcome after closed functional treatment of humeral shaft fractures. J Orthop Trauma. 2006;20(9):591-6.

33. Ring D, Chin K, Taghinia AH, Jupiter JB. Nonunion after functional brace treatment of diaphyseal humerus fractures. J Trauma. 2007;62(5):1157-8. 